The Role of Metacognitive Beliefs and Mathematical Self-Esteem in Predicting the Mathematical Competence of Preschool Children

\title{
Hamed Salehi
}

Allameh Tabatabaei University, Tehran, Iran

\section{Article Info:}

\section{ABSTRACT}

Introduction: Metacognition and self-concept are considered as one of the cognitive strategies in the mathematical competence of preschool children. The purpose of this study was to investigate the relationship between metacognitive beliefs, mathematical self-concept, and mathematical competence of preschool children. Materials and Methods: This study was a descriptive correlational. The statistical population of this study was preschool children of Tehran, Iran in the academic year 2019-2020. 200 children were selected and randomly selected according to Morgan's table. Utrecht's mathematical competence, Salonen et al.'s metacognitive beliefs and Marsh's mathematical self-concept were used to collect the data. Results: Our data have shown that metacognitive beliefs and mathematical self-concept have a positive and significant relationship with the mathematical competence of preschool children. Furthermore, mathematical self-concept can predict changes in the mathematical competence of preschool children. Conclusion: Attention to metacognitive beliefs and selfconcept play an important role in children's mathematical competence.

\section{*Corresponding Author: Hamed Salehi}

E-mail: salehi32.nm@gmail.com

Keywords:

1. Metacognition

2. Child

3. Attention 
نقش باورهاى فراشناختى و خودينداشت رياضى در بِيشبينى شايستكى رياضى كودكان بِيشدبستانى

حامد صالحىى

دانشعاه علامه طباطبايى، تهران، ايران

\section{اطلاعات مقاله:}

يذيرش: · r خرداد و جس

اصلاحيه: || |رديبهشت وجات مله:

دريافت: ه فروردين وج|

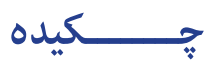

مقدمه: فراشناخت و خودينداشت به عنوان يكى از راهبردهاى شناختى در شايستخى رياضى كود كان

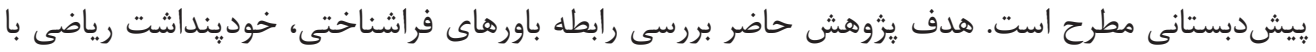

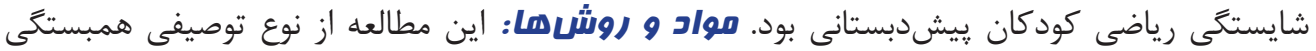

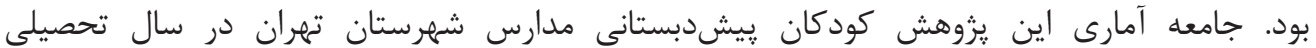

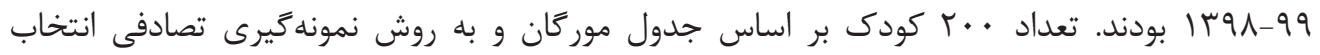

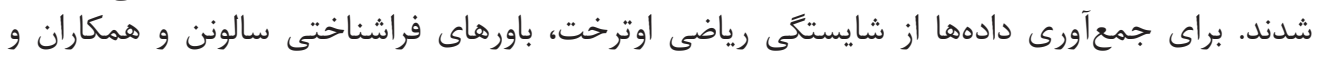

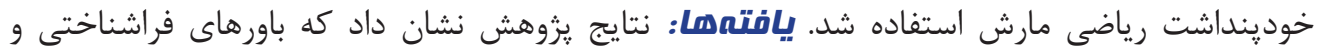

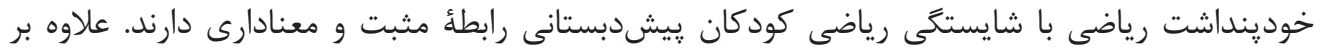

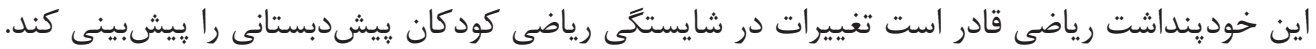

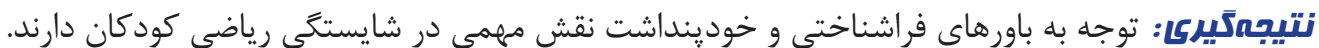

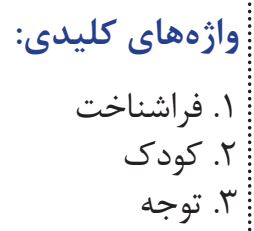

*نويسنده مسئول: حامد صالحى salehi32.nm@gmail.com يست الكترونيك 


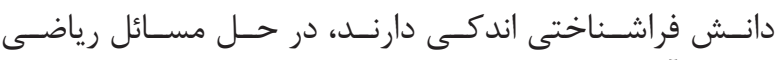

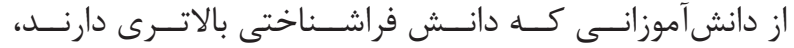

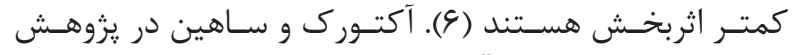

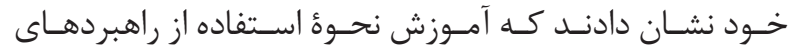

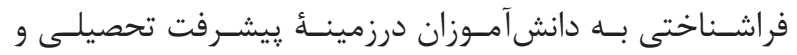

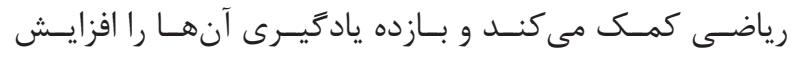

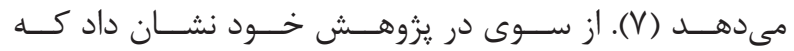

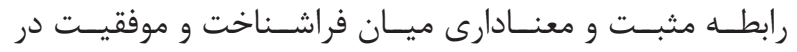

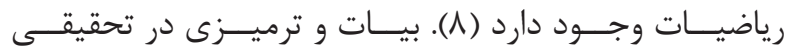

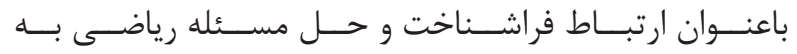

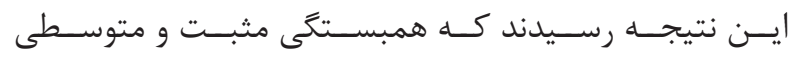

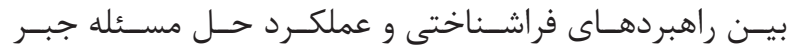

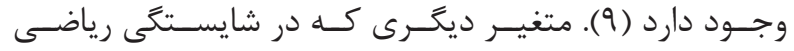

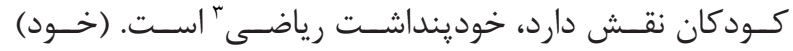

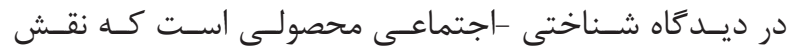

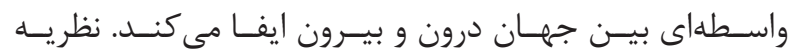

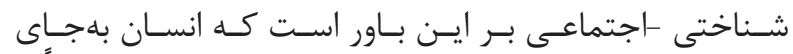

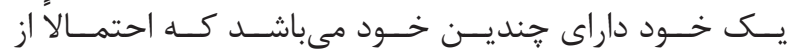

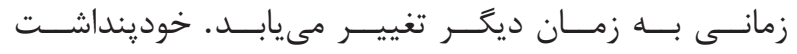

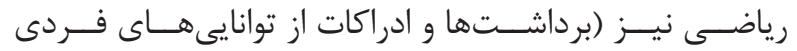

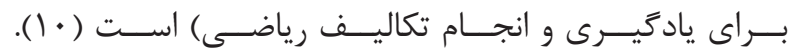

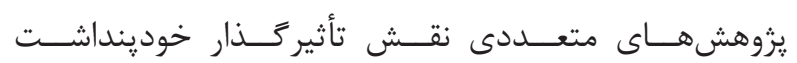

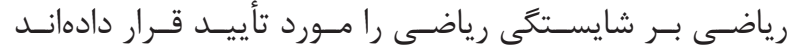

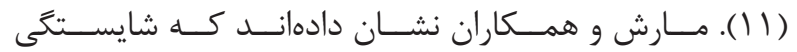

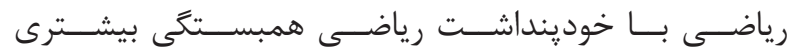

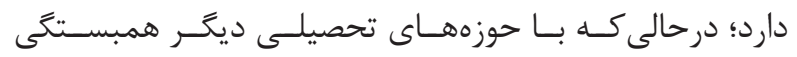

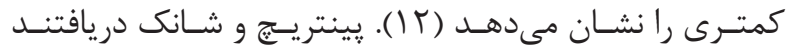

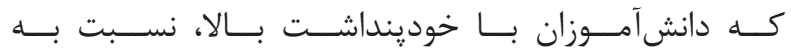

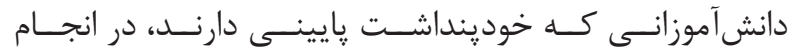

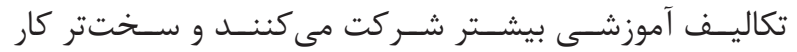

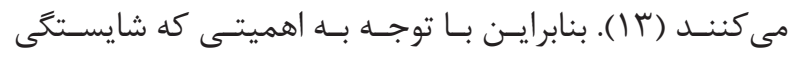

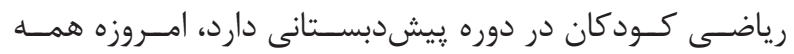

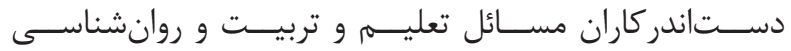

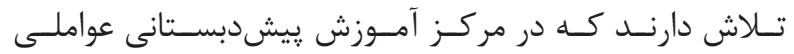

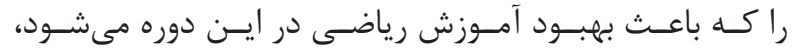

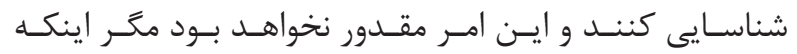

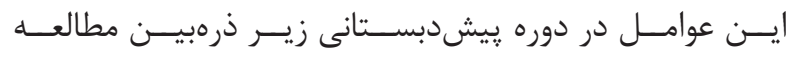

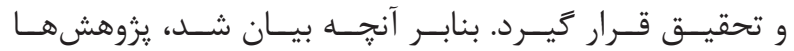

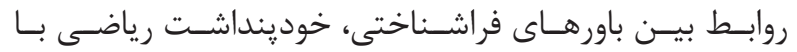

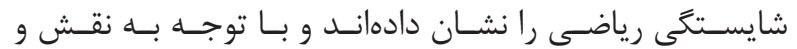

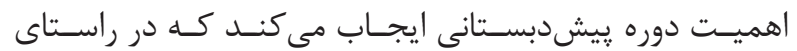

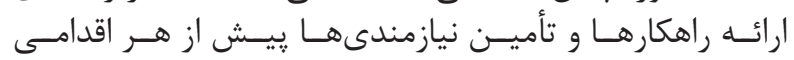

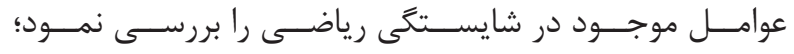

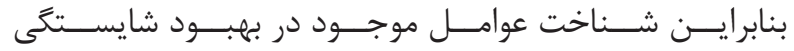

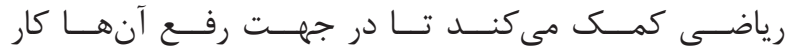

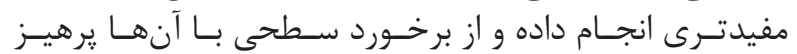

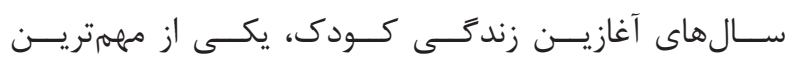

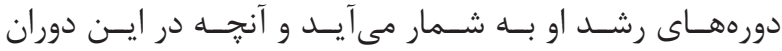

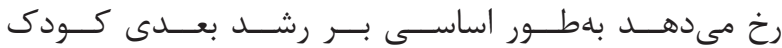

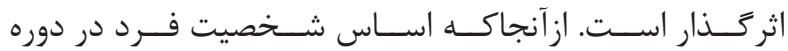

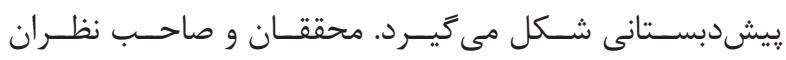

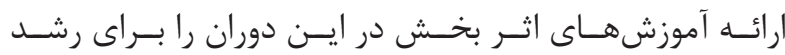

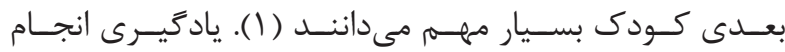

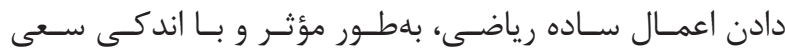

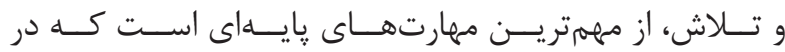

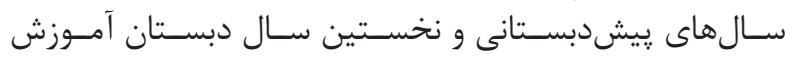

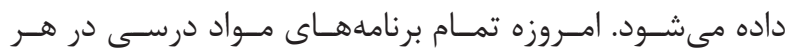

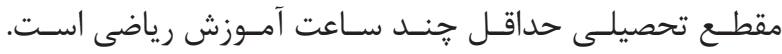

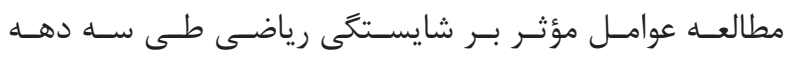

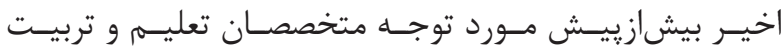

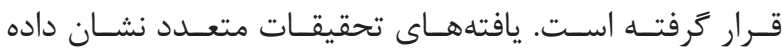

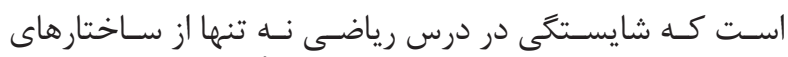

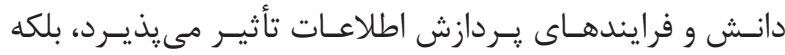

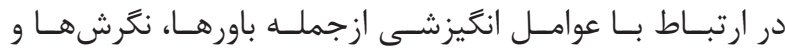

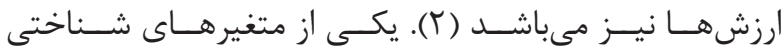

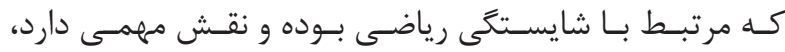

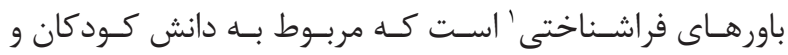

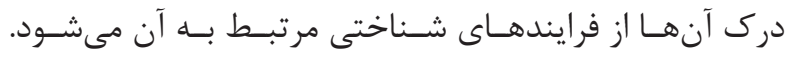

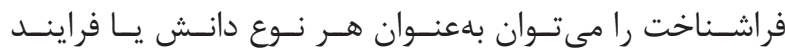

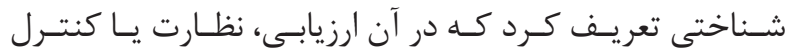

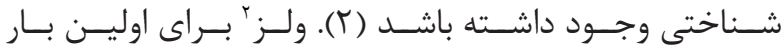

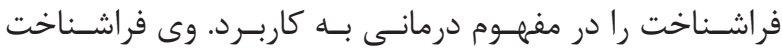

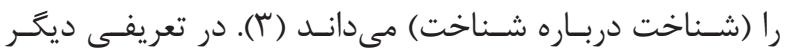

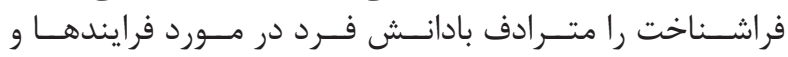

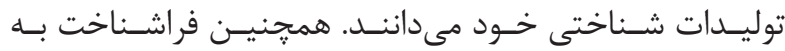

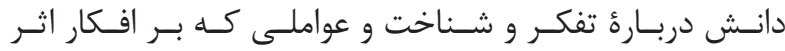

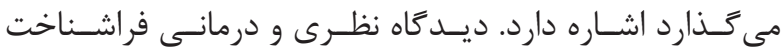

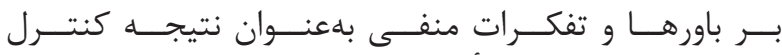

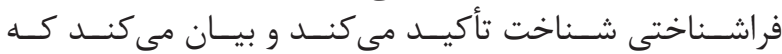

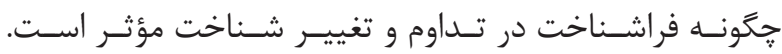

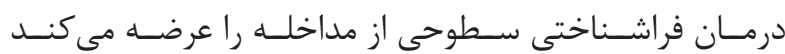

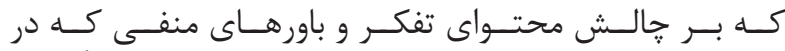

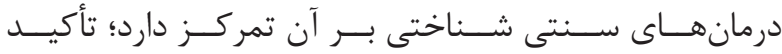

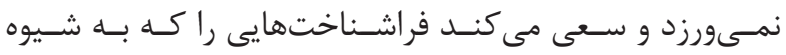

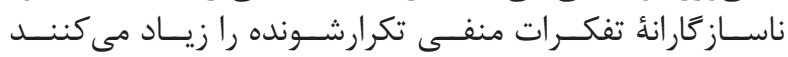

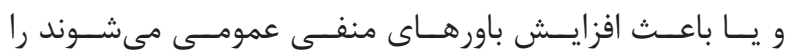

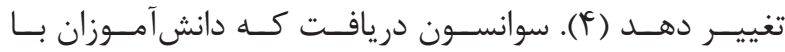

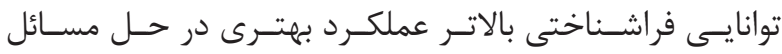

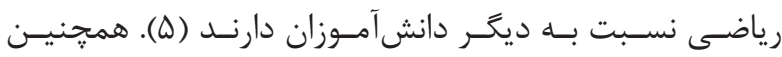

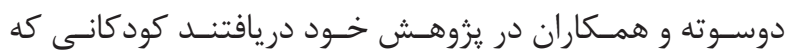




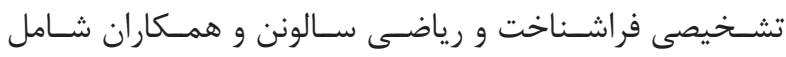

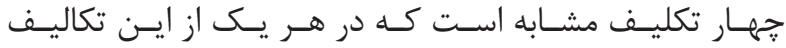

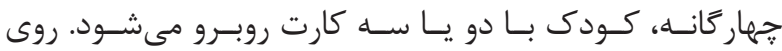

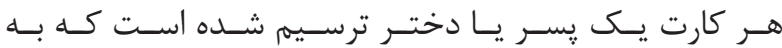

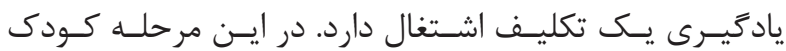

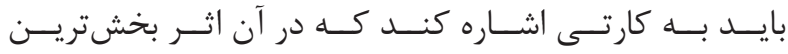

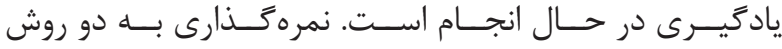

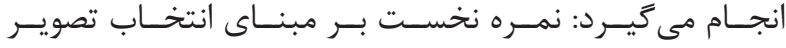

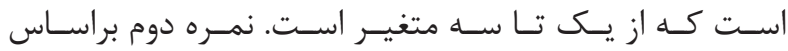

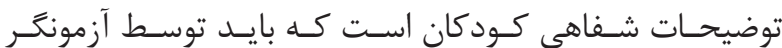

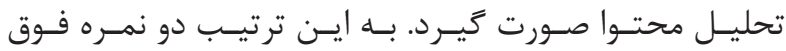

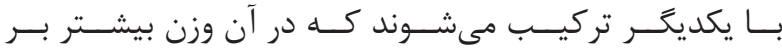

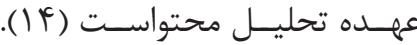
ج: آزمون خود ينداشت رياضى

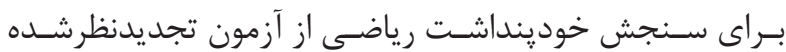

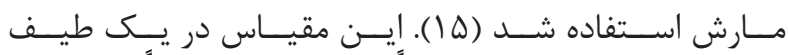

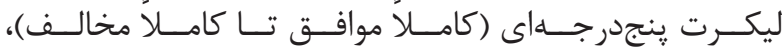

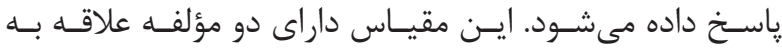

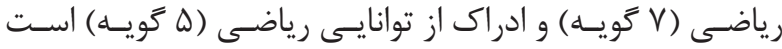

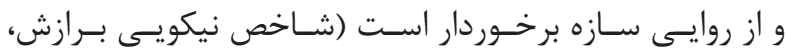

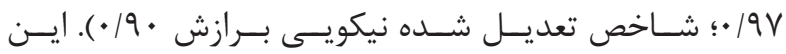

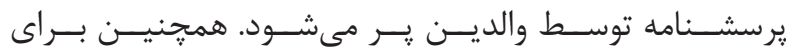

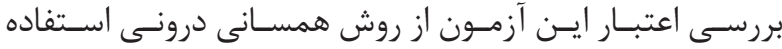

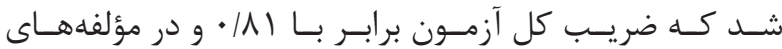

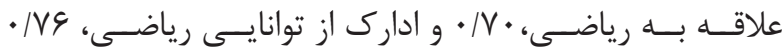

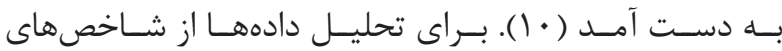

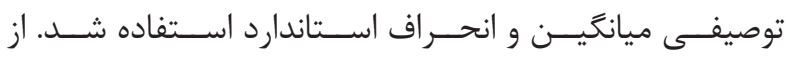

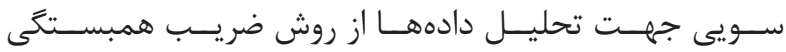

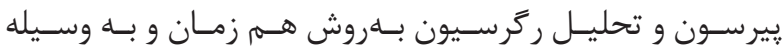

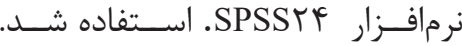

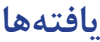

در جــدول آدارههــاى توصيفــى متغيرهــاى يزوهــش

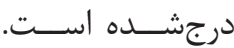

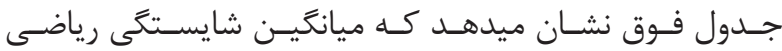

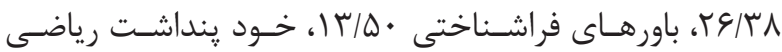

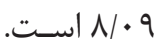

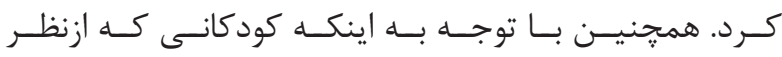

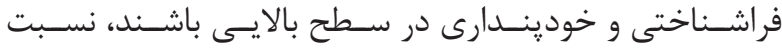

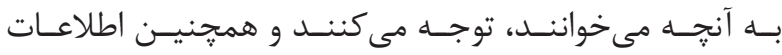

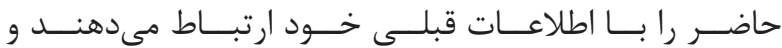

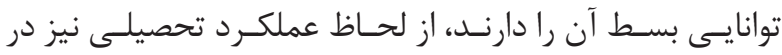

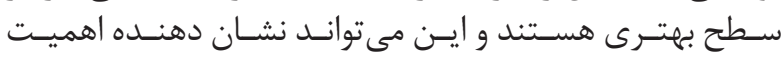

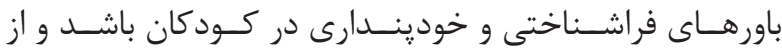

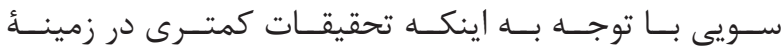

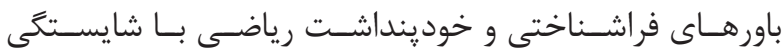

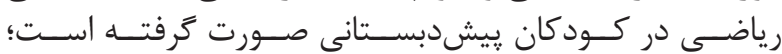

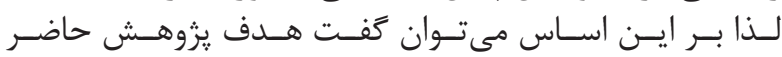

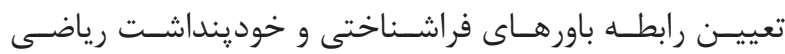

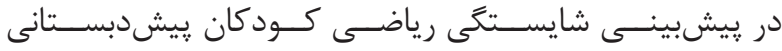

بـود.

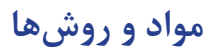

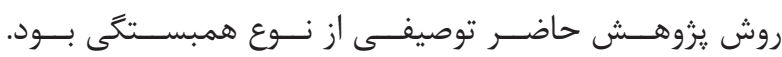

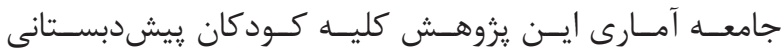

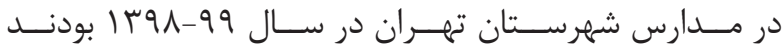

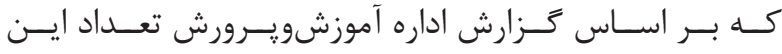

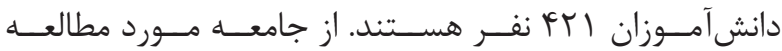

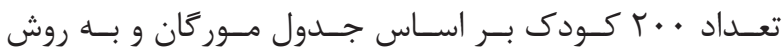

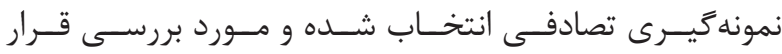

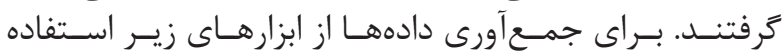
شــد:

\section{الف: مقياس شايستخَى رياضى كودكان اوترخت}

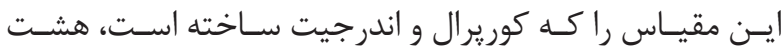

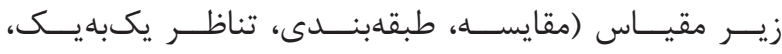

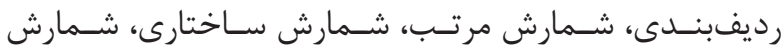

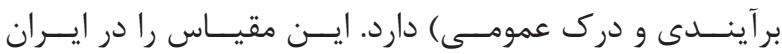

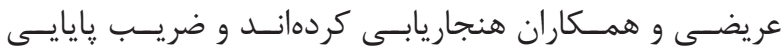

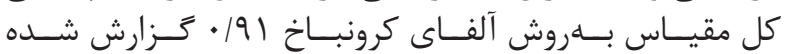

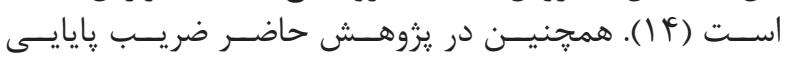

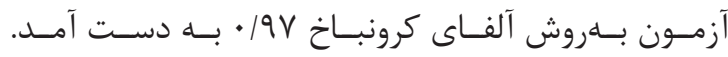

$$
\text { ب: آزمون باورهاى فراشناختى لـ آن }
$$

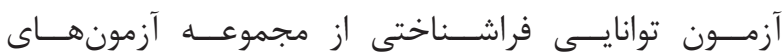

\begin{tabular}{|c|c|c|c|}
\hline انحر اف استاندارد & ميانگين & 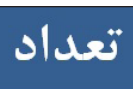 & متغير \\
\hline V/Ar & rG/r & $r \ldots$ & شايستخى رياضى \\
\hline $\boldsymbol{F} / \wedge \wedge$ & • & r.. & باورهاى فراشناختى \\
\hline F/g & $N / \cdot q$ & $r$. & خود ينداشت رياضى \\
\hline
\end{tabular}




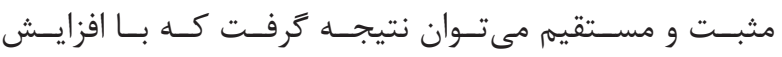

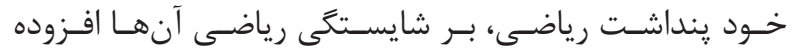

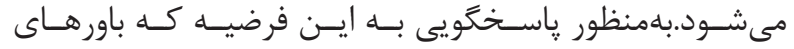

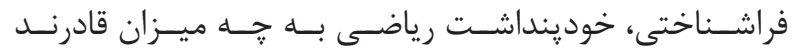

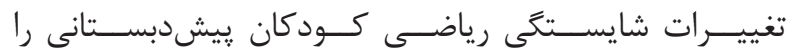

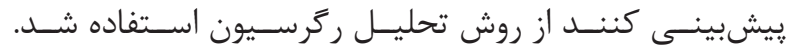

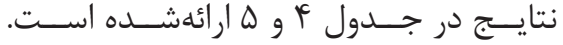

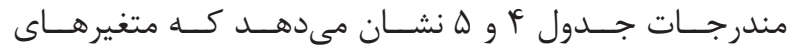

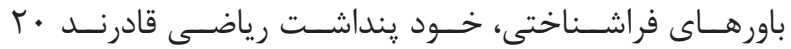

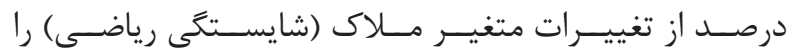

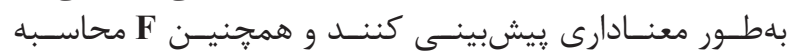

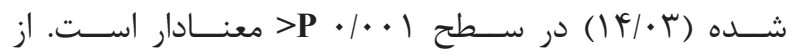

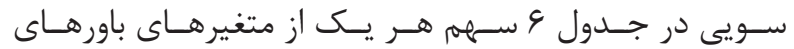

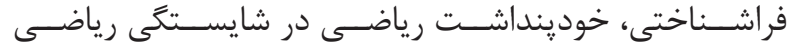

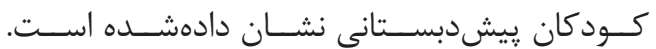

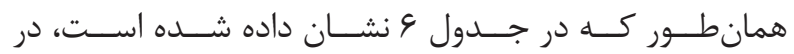

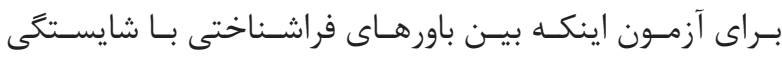

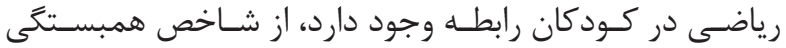

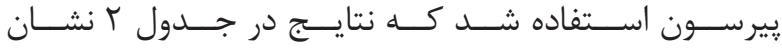

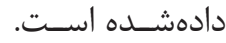

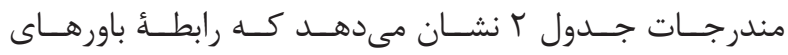

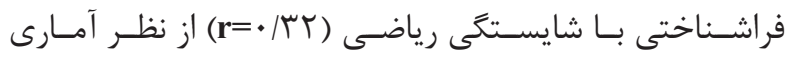

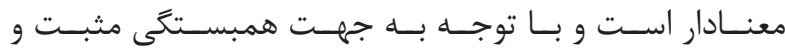

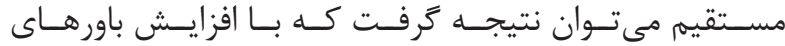

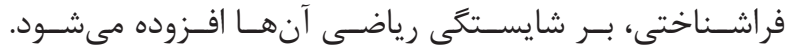

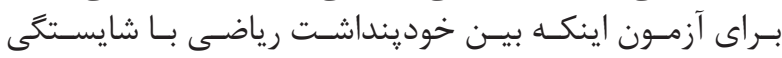

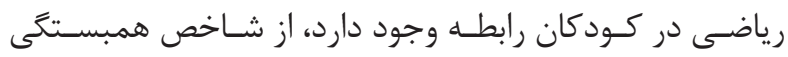

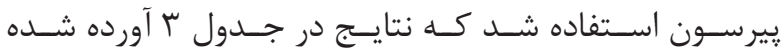
استـا

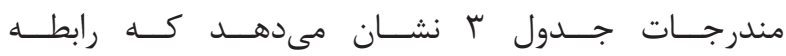

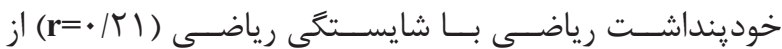

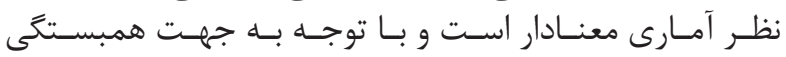

\begin{tabular}{|c|c|}
\hline شايستعَى رياضى & متغير \\
\hline $\begin{array}{c}r=\cdot / r r \\
p=\cdot / \cdot r\end{array}$ & باورهاى فر اشـناختى \\
\hline
\end{tabular}

جدول بـ- آزمون همبستخى بين خود بنداشت رياضى با شايستى رياضى

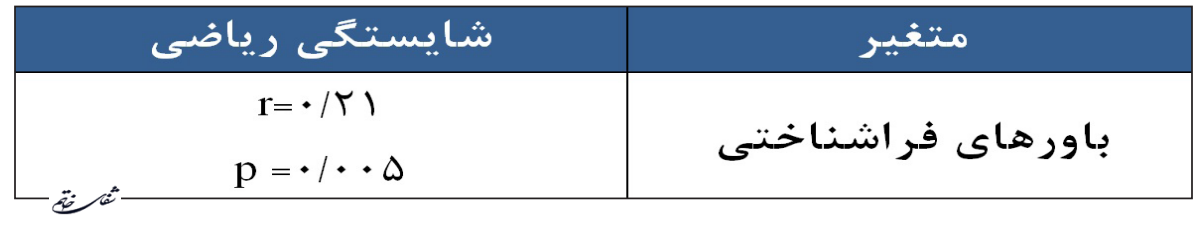

جدول f: خلاصه يافتهاى حاصل از تحليل ركر سيون به روش همزمان براى بيشبينى شايستَى رياضى

\begin{tabular}{|c|c|c|c|}
\hline خطاي استاندارد ميانتين & $\mathrm{R}^{2}$ & $\mathrm{R}$ & ملدل \\
\hline - & $\cdot \pi$. & 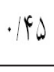 & هميزمن \\
\hline
\end{tabular}

\begin{tabular}{|c|c|c|c|c|c|}
\hline سطح معنادارى & $\mathrm{F}$ & ميانكين مجنورات & درجه آزادى & مجموع مجنورات & ملدل \\
\hline & & $994 / 11$ & $r$ & $r \cdot \Lambda r / 9 \varphi$ & ركّرسيون \\
\hline$\cdot \cdot \cdot 1$ & $\mid r / r$ & $\kappa q /<q$ & 109 & $V \wedge \notin 9 / V D$ & باقيمانده \\
\hline & & & 194 & $99 \Delta \psi / 4)$ & كل \\
\hline
\end{tabular}

\begin{tabular}{|c|c|c|c|c|c|}
\hline سطح معنادارى & $\mathbf{t}$ & B & خطاى استاندارد & B & مدل \\
\hline$\cdot 1+\cdots 1$ & $4 / 99$ & & T/TV & $11 / 1 V$ & ضريب ثابت \\
\hline$\cdot 1 \cdots 1$ & $r / \mathrm{KO}^{\prime}$ & • & $.11 \mathrm{r}$ &.$/ 4 t$ & باورهاى فراشناختى \\
\hline$\theta+1 \cdot t$ & $r / l f$ & .179 & .1 .9 & .149 & خود بنداشت رياضى \\
\hline
\end{tabular}




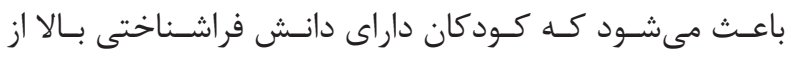

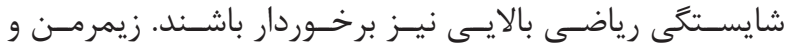

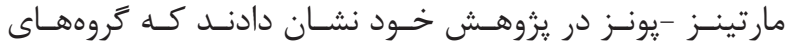

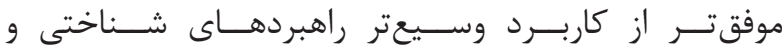

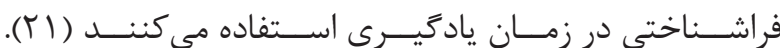

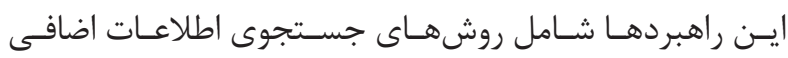

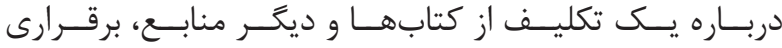

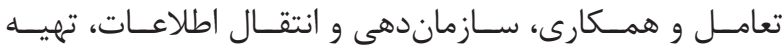

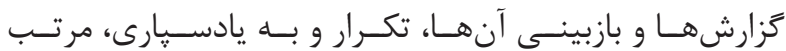

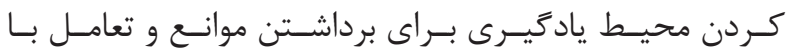

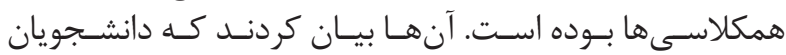

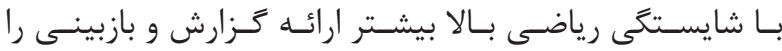

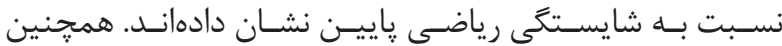

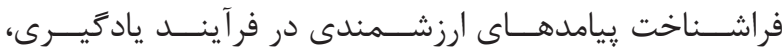

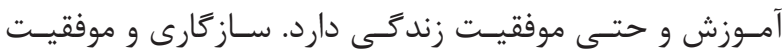

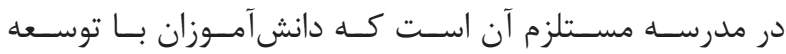

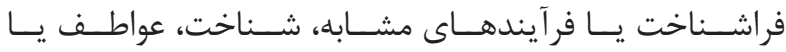

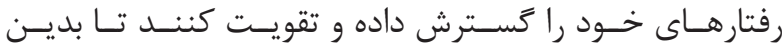

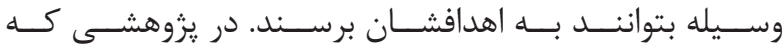

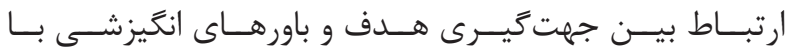

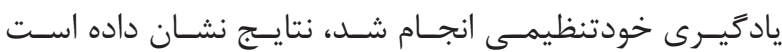

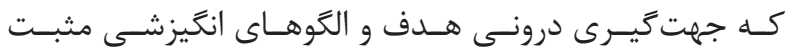

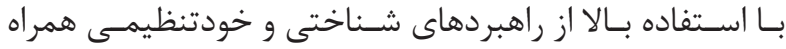

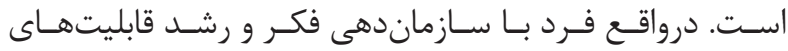

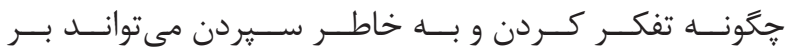

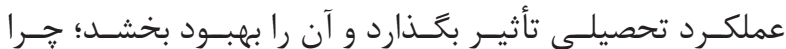

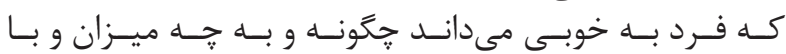

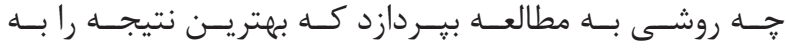

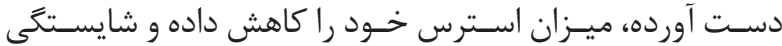

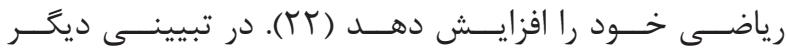

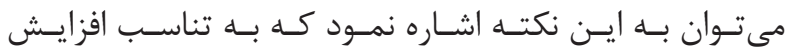

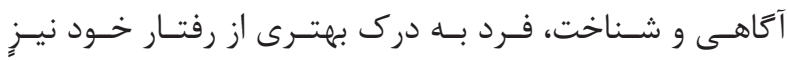

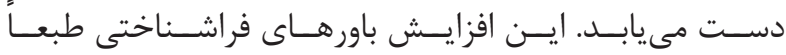

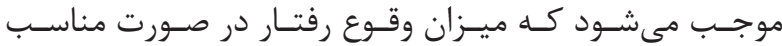

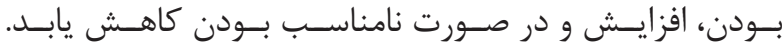

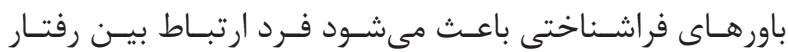

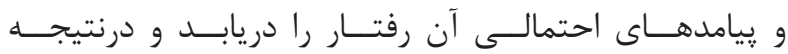

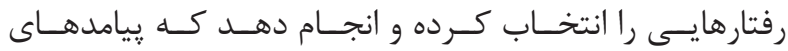

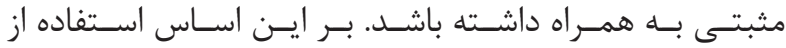

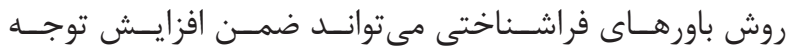

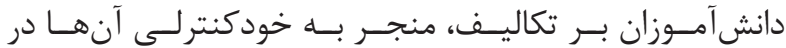

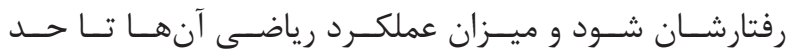

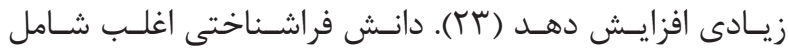

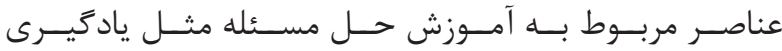

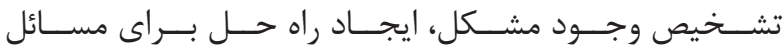

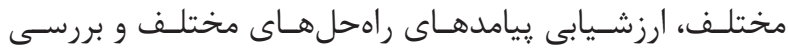

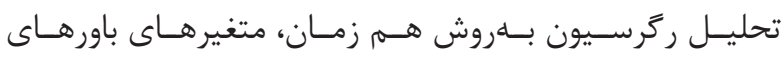

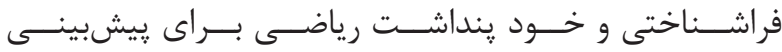

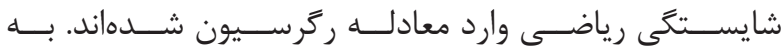

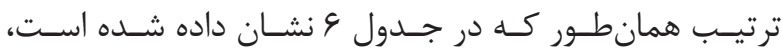

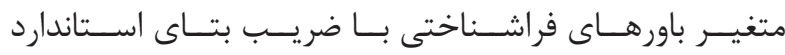
r • ن

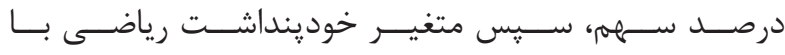

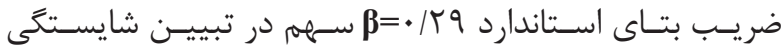

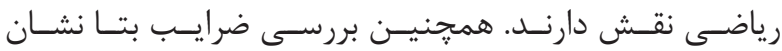

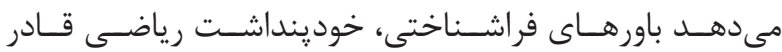

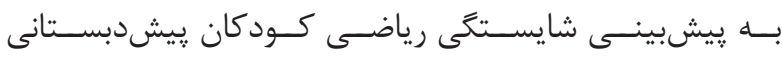

بحث و نتيجه كيرى

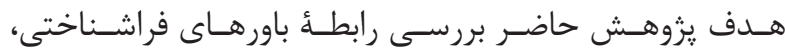

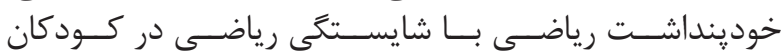

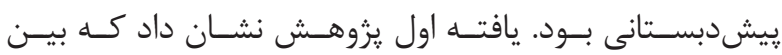

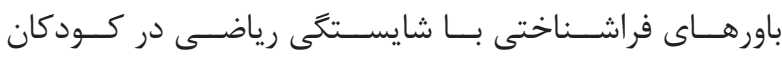

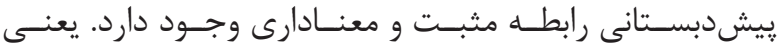

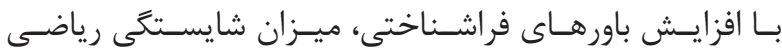

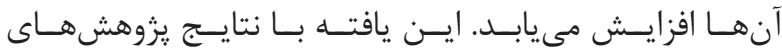

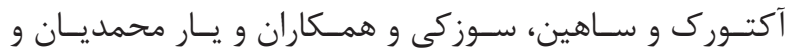

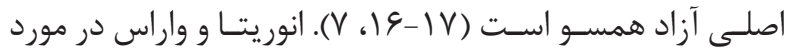

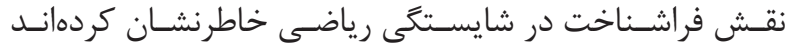

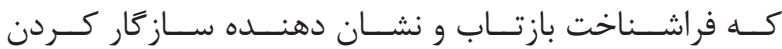

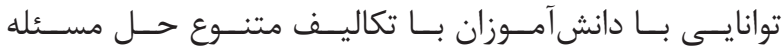

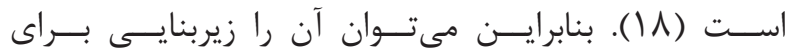

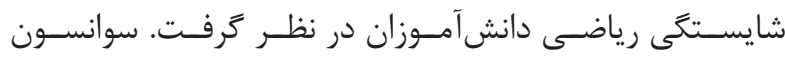

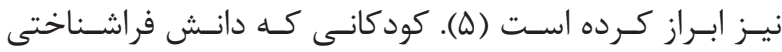

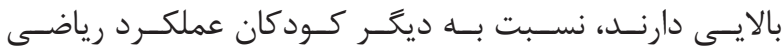

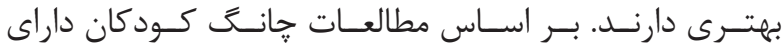

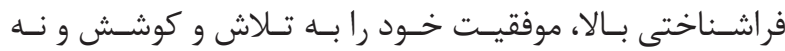

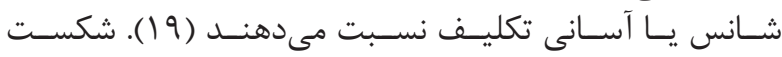

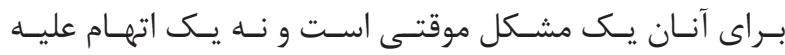

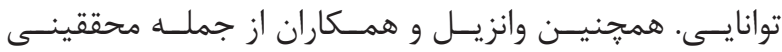

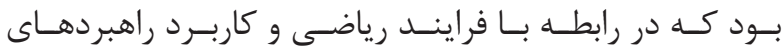

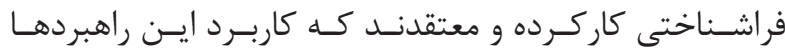

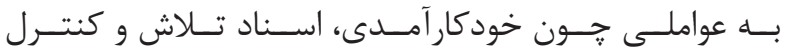

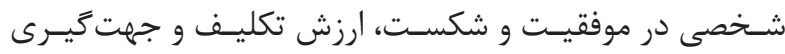

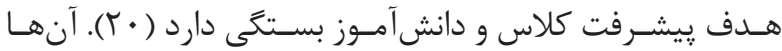

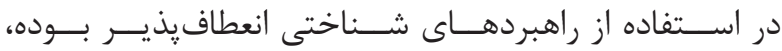

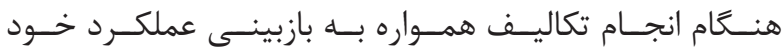

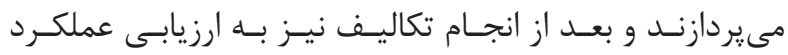

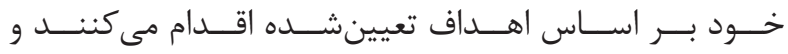

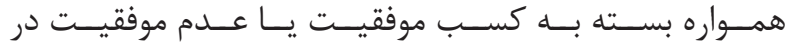

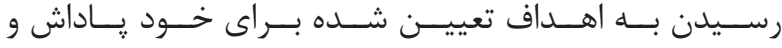

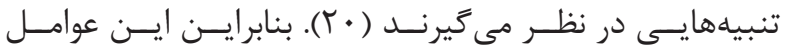




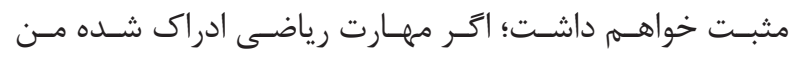

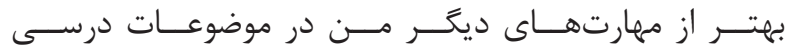

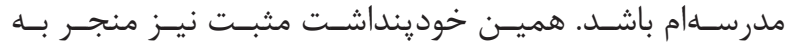

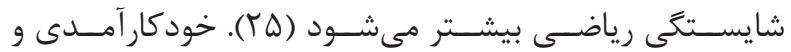

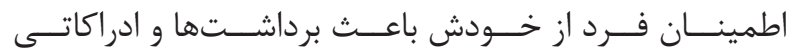

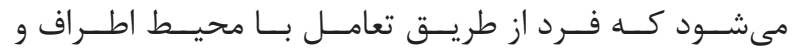

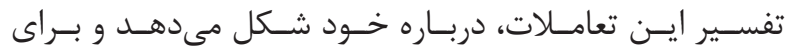

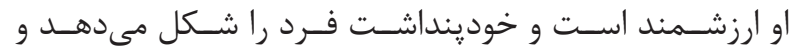

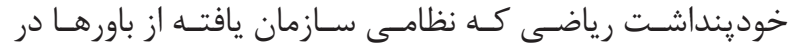

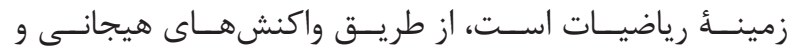

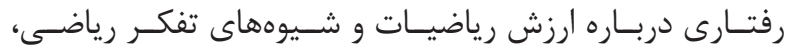

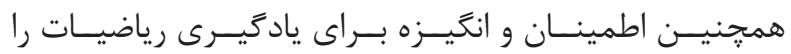

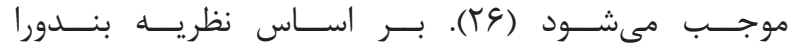

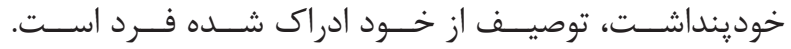

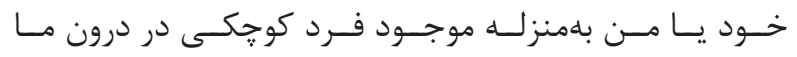

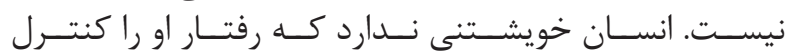

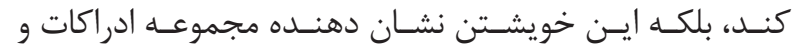

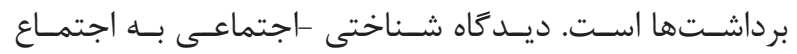

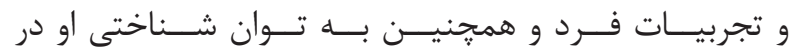

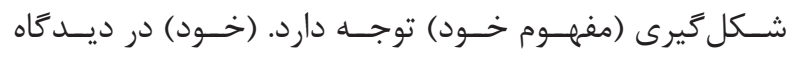

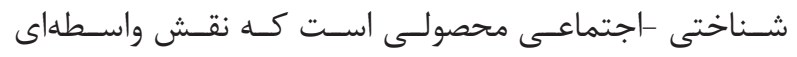

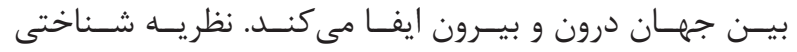

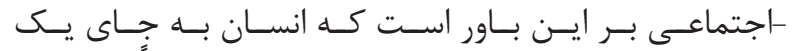

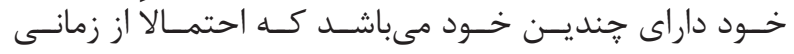

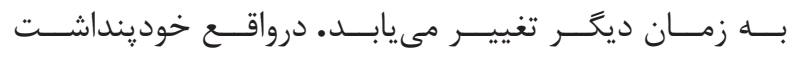

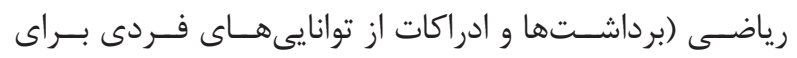

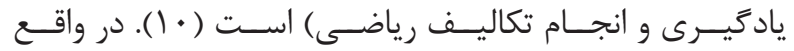

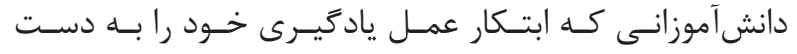

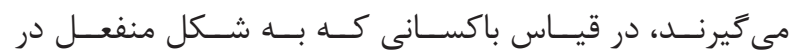

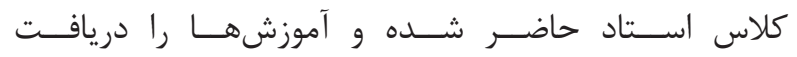

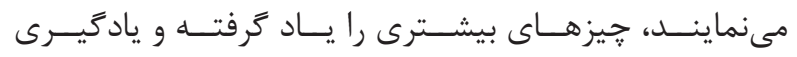

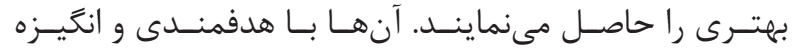

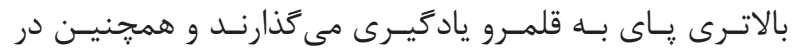

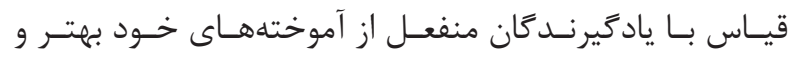

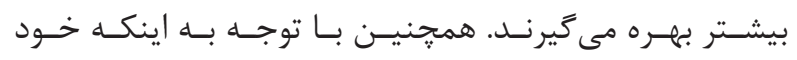

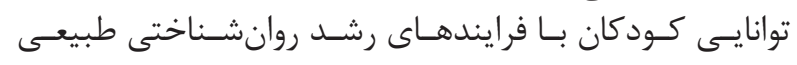

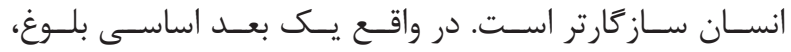

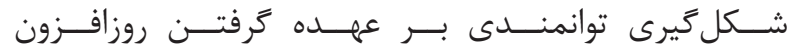

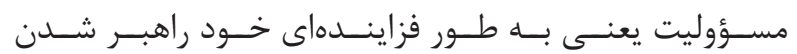

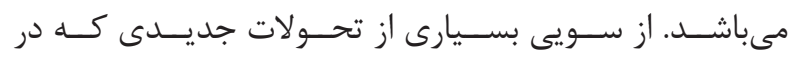

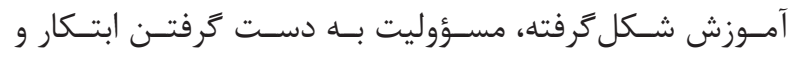

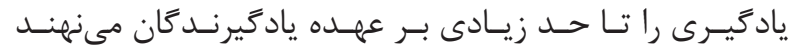

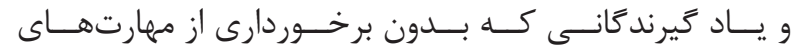

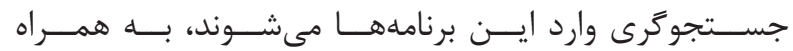

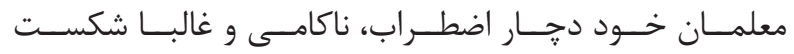

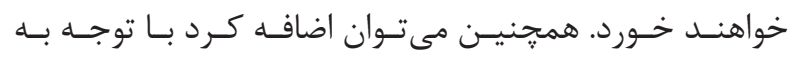

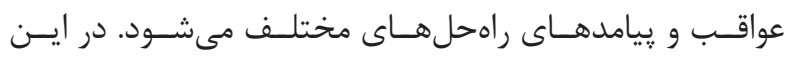

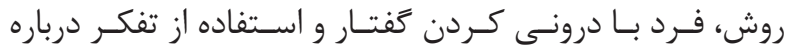

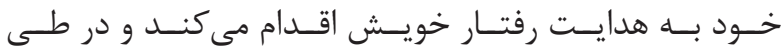

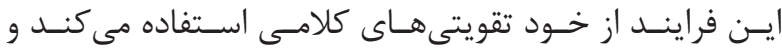

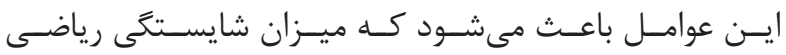

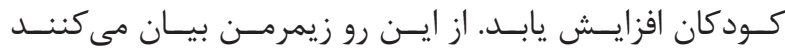

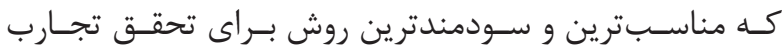

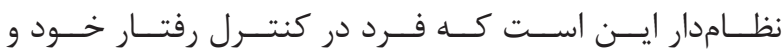

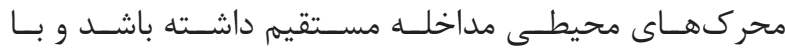

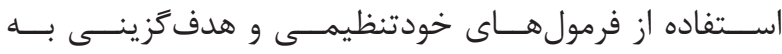

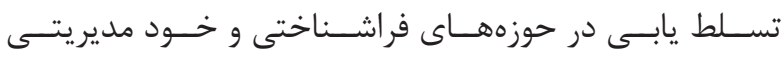

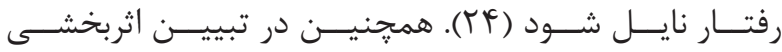

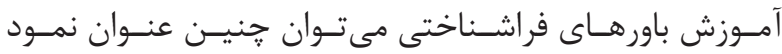

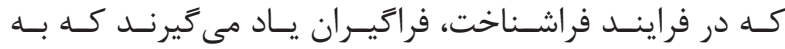

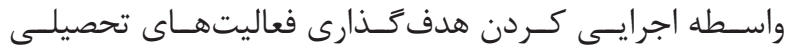

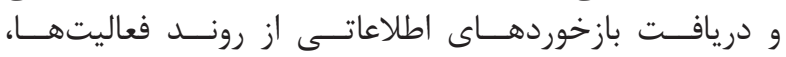

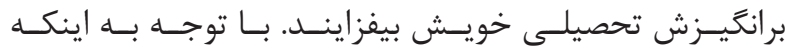

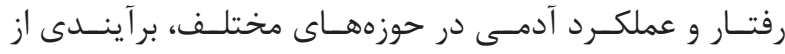

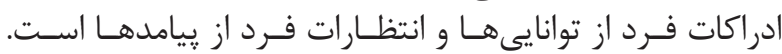
ايـن ادراكات و انتظــارات بــدون وسـاطت تجربــهـ مســقتيمى و

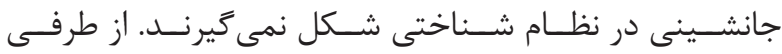

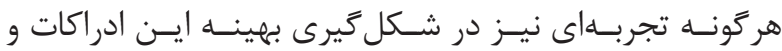

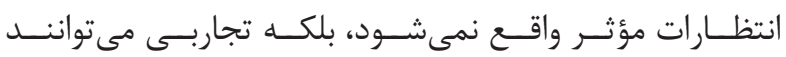

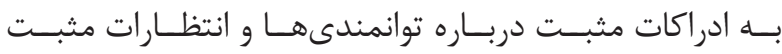

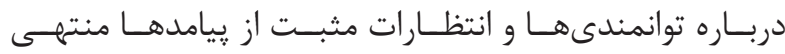

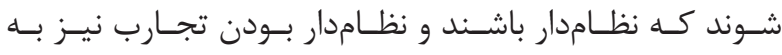

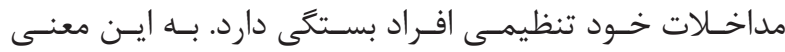

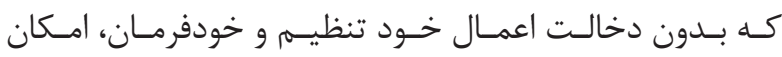

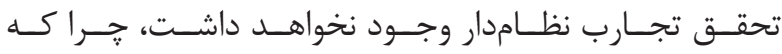

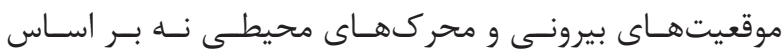

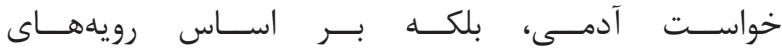

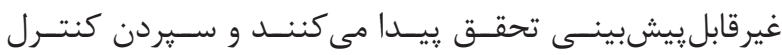

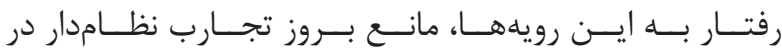

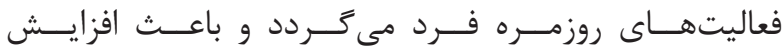

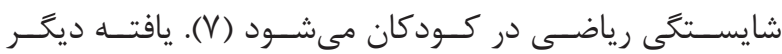

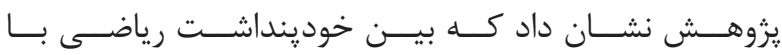

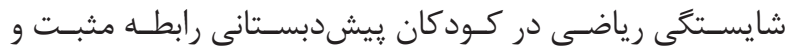

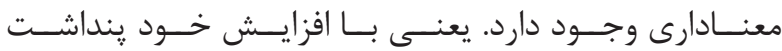

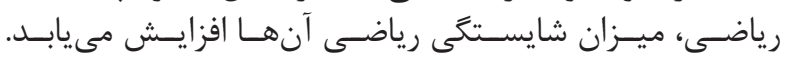

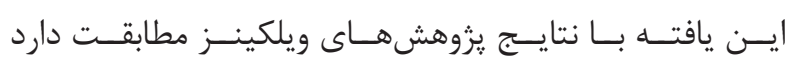

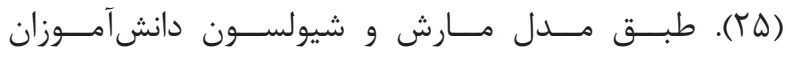

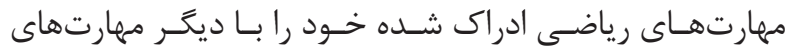

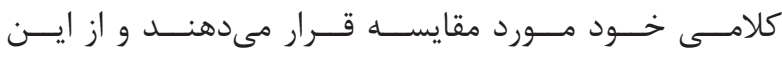

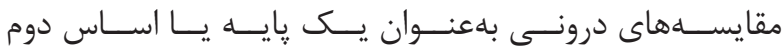

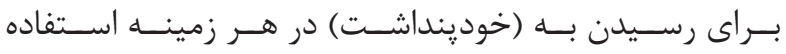

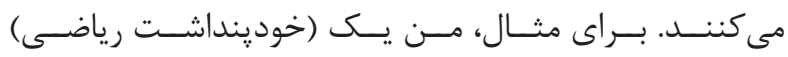




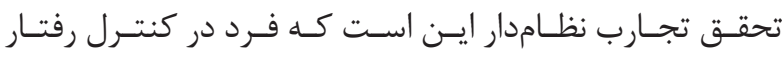

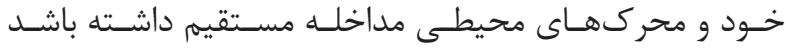

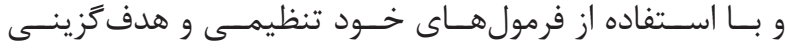

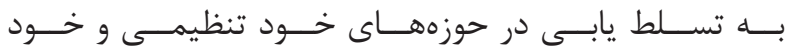

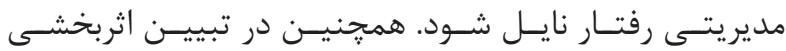

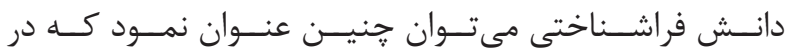

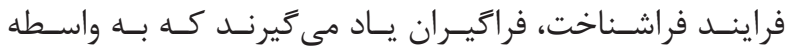

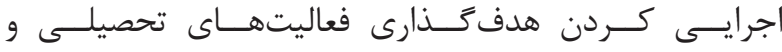

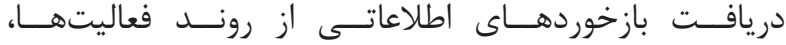

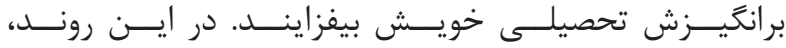

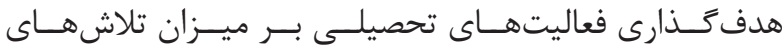

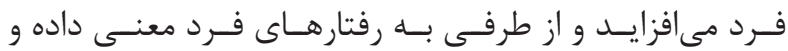

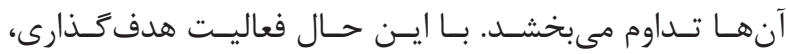

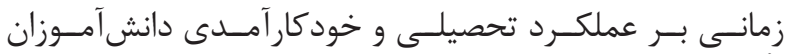

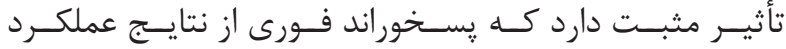

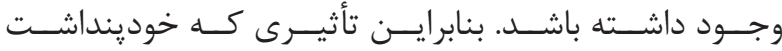

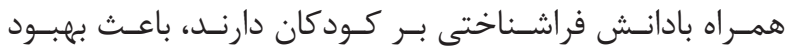

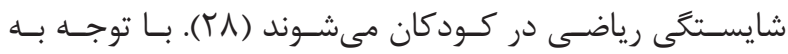

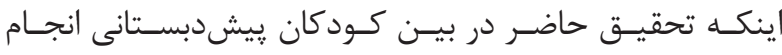

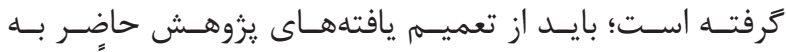

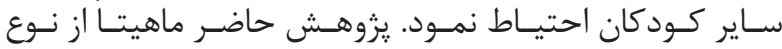

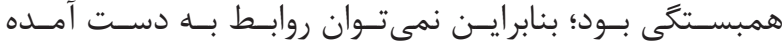

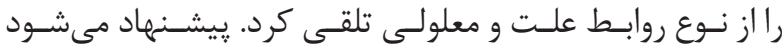

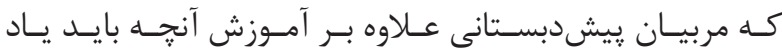

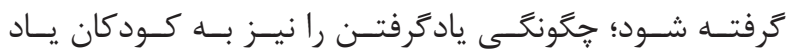

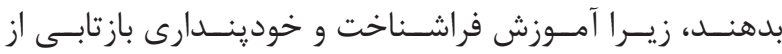

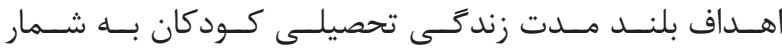

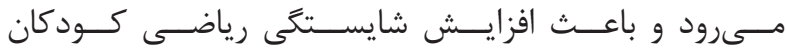

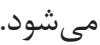

1. Barkhardari, M Jamshidian A. Citizenship education. Tehran: Academic Jihad Publications; 2008.

2. Shams F, Tabebordbar F. Mediating role of academic self-efficacy for goal oriantation and math performance. Psychological methods and models 2011; 3(1): 82-95.

3. Wells A. Emotional disorders and metacognition: Innovative cognitive therapy. Chichester: Wiley; 2000.

4. Wells A, Sembi S. Metacognitive therapy for PTSD: a preliminary investigation of a new brief treatment. $\mathrm{J}$ Behav Ther Exp Psychiatry2004; 35(4):307-18.

5. Swanson LH, Jerman O. The influence of working memory of reading growth in subgroups of children with reading disabilities. Journal of Exceptional Child Psychology 2007; 96(4): 249- 83.

6. Desoete A, Ozsoy G. Introduction: Metacognition,

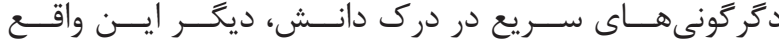

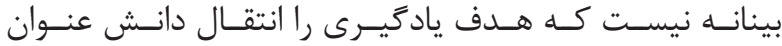

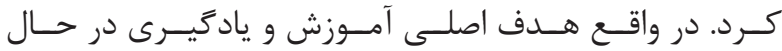

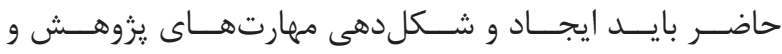

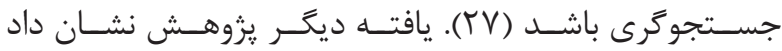

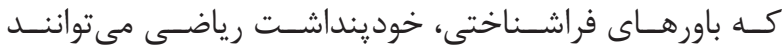

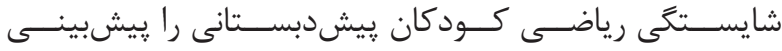

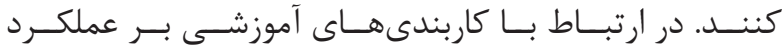

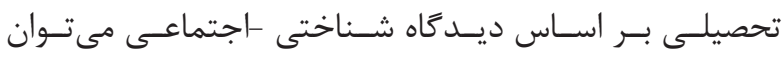

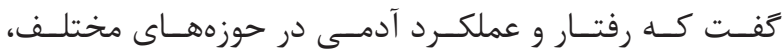

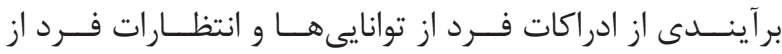

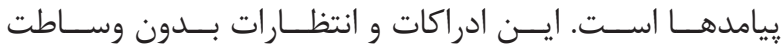

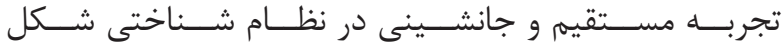

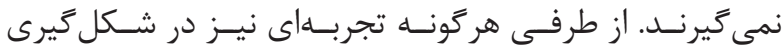

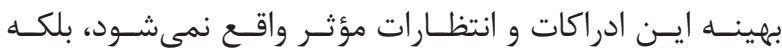

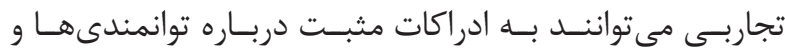

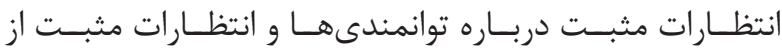

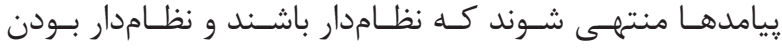

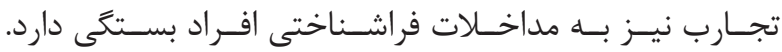

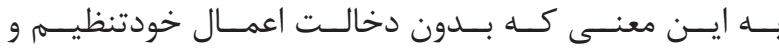

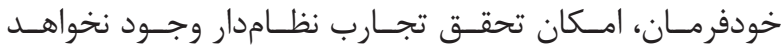

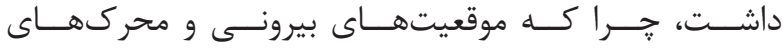

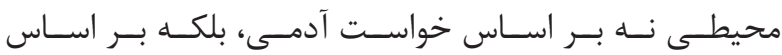

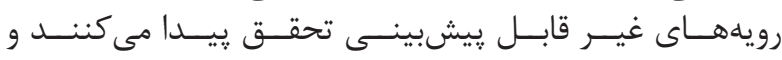

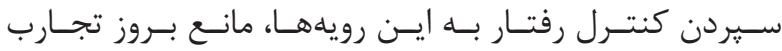

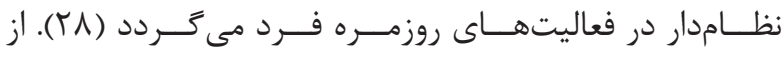

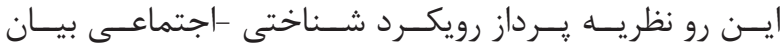

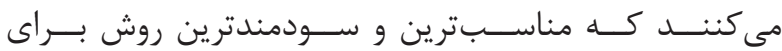

منابع more than the lognes monster? International Electronic Journal of Elementary Education 2009; 2(1): 1-6.

7. Akturk AO, Sahin I. Literature Review on Metacognition and its Measurement. Procedia social and behavioral sciences 2011; 15(3): 3731-736.

8. Ozsoy G. An investigation of the relationship between metacognition and mathematics achievement. Journal of Asia Pacific Education 2011; 12: 227-35.

9. Bayat S, Tarmizi RA. Assessing cognitive and metacognitive strategies during Algebra problem solving among university students. Procedia Social and Behavioral Sciences 2010; 8(3): 403-10.

10. Sharifi saki, Sh, Falah MH, Zare H. Role of mathematics self-efficacy, mathematics self-concept and perceived classroom environment in students> mathematics achievement with controlling the gender 


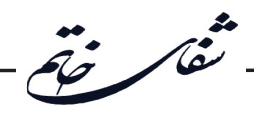

role. Quarterly Journal of Research in School and Virtual Learning 2014; 7(3): 9-22.

11. Anjum R. The Impact of self-efficacy on Mathematics Achievement of primary school children.Pakistan Journal of psychological Research 2006; 21: 61-78.

12. Marsh HW, Byrne BM, Yeung AS. Causal ordering of academic selfconcept and achievement: reanalysis of a pioneering study and revised recommend-ations. Educational Psychologist 1997; 34: 154-57.

13. Pintrich PR, Schunk D. Motivation in education: theory, research, and applications (2nd ed). Upper Saddle River, New Jersey: Prentice-Hall; 2002.

14. Oreizi HR, Abedi A, Taji M. Investigation the relation of counting capability, visualizing, hearing perception and meta-cognitive knowledge with mathematical competence in preschool children in the city of Isfahan. Educational Innovation 2008, 13(4); 132-48.

15. Marsh HW. Self-concept theory, measurement and research into practice: The role of self-concept in educational psychology. Leicester, UK: British Psychological Society; 2007.

16. Suzuki I, yanagi H, Tomura S. A study of factors related of activities of daily living (ADL) of the elderly receiving in home service longitudinal study using functional independence measure. Nippon Koshu Eisei Zasshi. 2007; 54(2): 81-88.

17. Yarmohammadian A, Asli-Azad M. Effects of Metacognition Training on the Improvement of Mathematical Function in Children with Mathematic Learning Disability. Advances in Cognitive Sciences 2012; 14 (1):41-52.

18. Annevirta T, Vauras M. Metacognitive knowledege in primary grades: A longitudinal study. European Journal of Psychology of Education 2001; 16: 257-82.

19. Chang CY. A study of the relationship between college students academic performance and cognitive s tyle, metacognition, motivational and self-regulation factors. Educational psychology 1991; 24: 145-61.

20. Vanzile- Tamsen C, Lovigston JA. The differential impact of motivation on the self- regulated strategy use of high- and lo wachieving college students. Journal of collegesrudent development 2009; 40(1): 45-60.

21. Zimmerman BJ, Martinez- pons N. Student Differences in self- regulated learning: relating grade, sex and gifteduness to self- efficacy and strategy use journal of Educational psychology 1990; 84(1): 51-59.

22. Mousavi S, Jabalameli J, Alibakhshi F. Study of the relation emotional intelligence (EI) and, motivation beliefs self-regulatory on the academic performance. Behavioral Sciences Research 2012; 10(3): 178-92.

23. Hindshaw D. Attention deficit hyperactivity disorder children. Thousand Oaks, CA: sage; 1994.

24. Zimmerman BJ. Self-regulation involves more than metacognition: A social cognitive perspective. Educational Psychologist 1995; 30(4): 217-21.

25. Wilkins JLM. Mathematics and science self-concept: An international investigation. Journal of Experimental Education 2004; 72: 331-46.

26. Marsh HW, Craven RG. Reciprocal effects of selfconcept and performance from a multidimensional perspective: Beyond seductive pleasure and unidimensional perspectives. Perspectives on Psychological Science 2006; 1: 133-63.

27. Bembenutty H. Self- Regulation of Learning and Academic Delay of Grafication: Gender and Ethnic Difference among College students. Journal of advanced academics 2008; 18(4): 586-16.

28. Bandura A. Much ado over a faulty conception of perceived self-efficacy grounded in faulty experimentation. Journal of Social and Clinical Psychology 2007; 26(6): 641-58. 